\title{
Increased levels and reduced catabolism of asymmetric and symmetric dimethylarginines in pulmonary hypertension Soni Pullamsetti ${ }^{* 1}$, Ladislau Kiss ${ }^{1}$, Hossein Ardeschir Ghofrani ${ }^{1}$, Robert Voswinckel ${ }^{1}$, Peter Haredza ${ }^{1}$, Walter Klepetko ${ }^{2}$, Clemens Aigner ${ }^{2}$, Ludger Fink ${ }^{3}$, Jai Prakash Muyal ${ }^{3}$, Norbert Weissmann ${ }^{1}$, Friedrich Grimminger ${ }^{1}$, Werner Seeger ${ }^{1}$ and Ralph Theo Schermuly ${ }^{1}$
}

Address: ${ }^{1}$ Department of Internal Medicine, Justus-Liebig-University Giessen, 35392 Giessen, Germany, ${ }^{2}$ Department of Cardiothoracic Surgery, University of Vienna, A-1090 Vienna, Austria and ${ }^{3}$ Department of Pathology, Justus-Liebig-University Giessen, 35392 Giessen, Germany

Email: Soni Pullamsetti* - Soni.Pullamsetti@innere.med.uni-giessen.de

* Corresponding author

from 2nd International Conference of cGMP Generators, Effectors and Therapeutic Implications

Potsdam, Germany, 10-12 June, 2005

Published: 16 June 2005

BMC Pharmacology 2005, 5(Suppl I):P45 doi:I0.| |86/I47|-22 I0-5-SI-P45

Asymmetric dimethylarginine (ADMA) is an endogenous inhibitor of nitric oxide synthase (NOS) and has been implicated in endothelial dysfunction. ADMA is metabolized by the enzyme dimethylarginine dimethylaminohydrolase (DDAH), with DDAH2 representing the predominant endothelial DDAH isoform. Symmetric dimethylarginine (SDMA), also originating from arginine methylation by protein arginine methyltransferases, is an inhibitor of intracellular arginine uptake. In both, chronic pulmonary hypertensive rats and patients suffering from idiopathic pulmonary arterial hypertension (IPAH; NYHA class III), a marked increase in plasma ADMA and SDMA levels as well as tissue levels of asymmetric and symmetric dimethylated proteins was observed. Moreover, when comparing lung tissue from pulmonary hypertensive rats and IPAH patients to corresponding normal lung tissue, expression of DDAH2 was found to be reduced at both, the mRNA and the protein level, with no significant changes in DDAH1 expression. These findings were further supported by demonstrating a decrease in DDAH2 function in the experimental pulmonary hypertension model. Immunohistochemistry in human and rat control tissue demonstrated both isoforms of DDAH in the endothelial layer and the in the alveolar epithelium. In contrast, in pulmonary hypertensive tissue, the immunoreactivity of DDAH2 in pulmonary endothelium was significantly decreased compared to DDAH1. Therefore, altogether we can conclude that enhanced dimethylarginine levels may contribute to vascular abnormalities in pulmonary arterial hypertension. Suppression of endothelial DDAH2 expression and function represent an important underlying mechanism. 